\title{
Clean Catch Urine Collection: Time taken and diagnostic implication. A Prospective Observational Study.
}

\section{Original Article}

Shidan Tosif, MBBS FRACP (1, 2, 3), Jonathan Kaufman MBBS (2, 3, 4), Patrick Fitzpatrick MB Bch BAO MRCPCH $(2,4)$, Sandy M Hopper MBBS FRACP FACEM (2, 3, 4, 5), Monsurul Hoq MSc (Statistics) MPH (6), Susan Donath PhD (3, 5), Franz E Babl MD MPH FRACP FAAP FACEP $(2,3,4)$
Affiliations
(1) Department of General Medicine, Royal Children's Hospital Melbourne
(2) Murdoch Children's Research Institute, Clinical Sciences
(3) Department of Paediatrics, University of Melbourne
(4) Emergency Department, Royal Children's Hospital Melbourne
(5) Emergency Department, Monash Medical Centre Melbourne
(6) Clinical Epidemiology and Biostatistics Unit, Murdoch Childrens Research Institute

\section{Address correspondence to: \\ Dr Shidan Tosif \\ Paediatrician \\ Department of General Medicine \\ Royal Children's Hospital \\ 50 Flemington Road \\ Parkville Victoria 3052 \\ Phone -61-3-9345-6160 \\ Fax -61-3-9345-5938 \\ Email shidan.tosif@,rch.org.au}

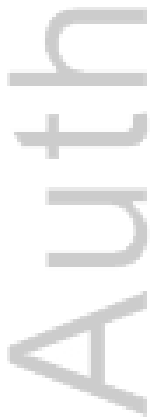

This is the author manuscript accepted for publication and has undergone full peer review but has not been through the copyediting, typesetting, pagination and proofreading process, which may lead to differences between this version and the Version of Record. Please cite this article as doi: 10.1111/jpc.13595

This article is protected by copyright. All rights reserved. 


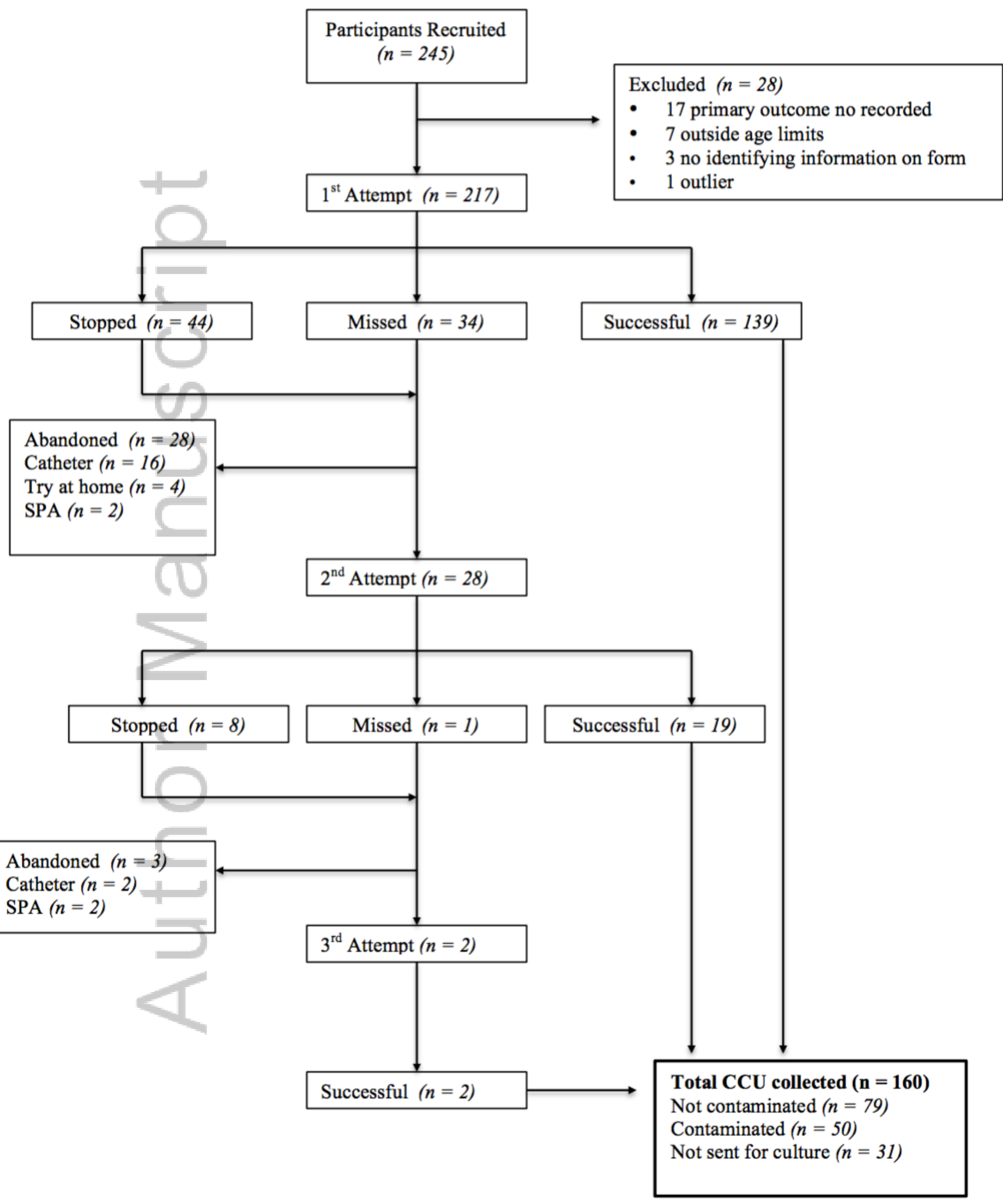

Figure 1.tiff

This article is protected by copyright. All rights reserved. 


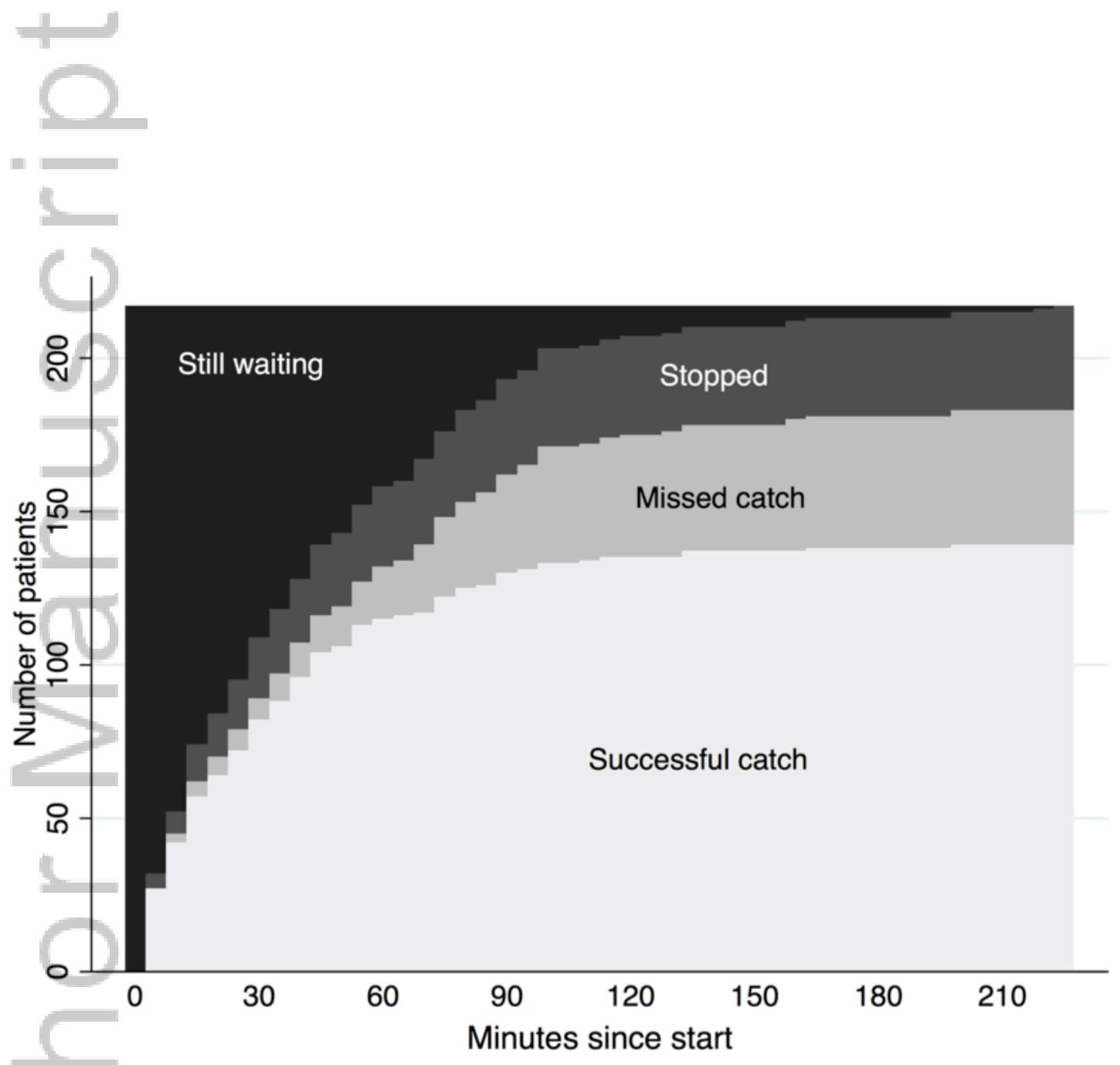

Figure 2.tiff

This article is protected by copyright. All rights reserved. 


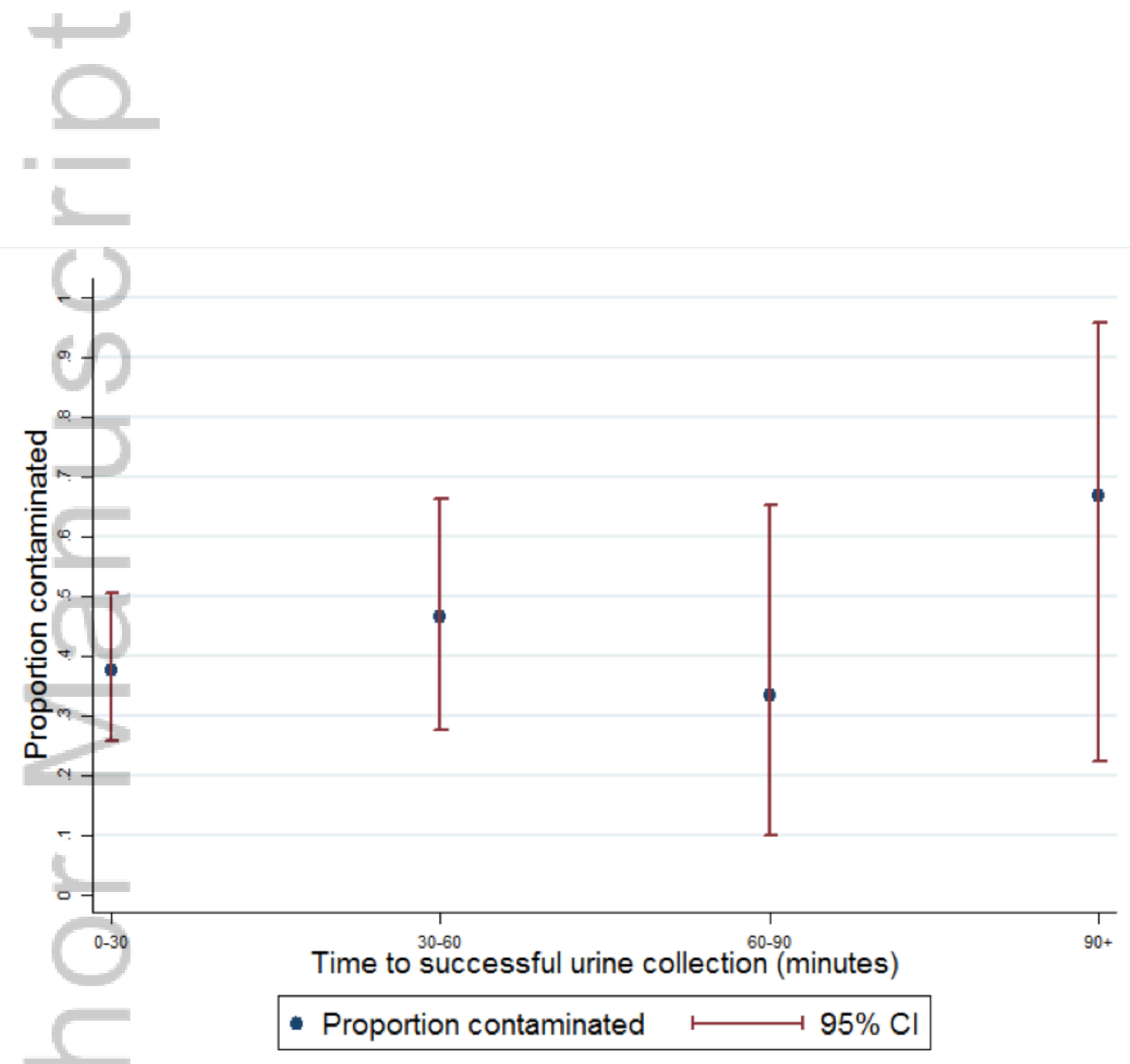

Figure 3.tiff

This article is protected by copyright. All rights reserved. 
Clean Catch Urine Collection: Time taken and diagnostic implication. A Prospective Observational Study.

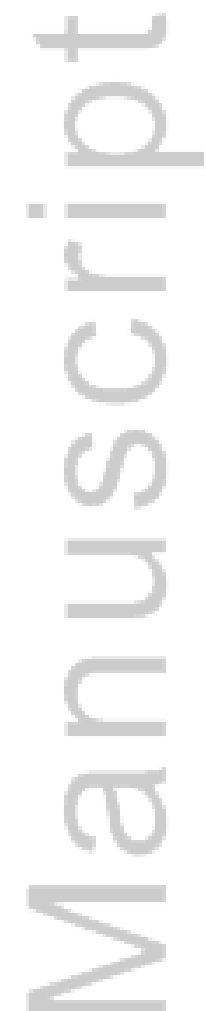

Short title: Clean catch urine attempt outcomes in young children

\author{
Abbreviations: \\ CCU clean catch urine \\ SPA suprapubic aspiration \\ CSU catheter specimen urine \\ CFU colony forming unit \\ UTI urinary tract infection \\ AAP American Academy of Pediatrics \\ NICE National Institute for Health and Care Excellence
}

This article is protected by copyright. All rights reserved. 


\section{What's Known on This Subject}

- Clean catch urine (CCU) is often the preferred method of urine specimen collection in pre-continent children, despite higher contamination rates than invasive methods.

- The time taken waiting for CCU sample collection is unclear

- Little is known about the relationship between CCU collection time and

$=$ contamination

\section{What This Study Adds}

- CCU attempts are time consuming

- CCU attempts have only a moderate success rate of obtaining a definitive sample

- Limitations of CCU are failure to void, missed catches and a high contamination rate
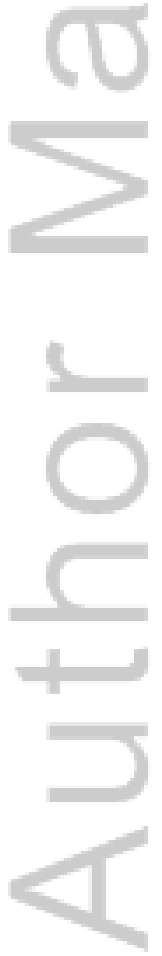

This article is protected by copyright. All rights reserved. 


\section{ABSTRACT}

BACKGROUND: Clean Catch Urine (CCU) collection is commonly used in precontinent children. CCU can be time-consuming and specimens may be contaminated. Our aim was to determine the time taken for CCU attempts and to describe the success of this method in diagnosing or excluding urinary tract infection.

METHODS: Prospective observational study of CCU in pre-continent children aged 2 48 months in the Emergency Department. Time taken until urine collection was "successful" (voided and caught), "missed" (voided not caught) or the procedure "stopped" was recorded and urine culture results analysed.

RESULTS: 217 children (131 [60\%] male) were enrolled. There were a total of 247 attempts at CCU. For the first attempt, the median collecting time was 30.5 minutes (IQR 11-66). Outcome was "successful" in 64\% (95\% CI 58 - 70\%), "missed" in 16\% (95\% CI $11-20 \%)$ and "stopped" in 20\% (95\% CI $15-26 \%)$. Median time if "successful" was 25 minutes (IQR 7 - 46.5), “missed” 27 minutes (IQR 11.6 - 59) and 71 minutes (IQR 42.5 - 93) when "stopped". 160 children had successful CCU collection, 129 were sent for culture. 50 of 129 (39\%) were contaminated. If all urine caught were sent for culture, the estimated yield of an uncontaminated urine specimen was $45 \%$. Contamination was not related to time taken for CCU.

CONCLUSIONS: CCU is time-consuming, frequently unsuccessful and contaminated, resulting in a low overall diagnostic yield. Clinicians could expect a $45 \%$ chance of obtaining a definitive urine sample from this method overall.

This article is protected by copyright. All rights reserved. 


\section{INTRODUCTION}

Urinary tract infections are common in children under 2 years of age, and affect between $5 \%$ and $7 \%$ of febrile children without a focus in this age group ${ }^{1-3}$. Obtaining a urine specimen is frequently required to diagnose or exclude urinary tract infection (UTI), and is recommended for the young child who looks unwell ${ }^{4,5}$, particularly infants under the age of 6 months $^{6}$.

A diagnosis of UTI requires a urine culture result of a sufficient bacterial colony count without evidence of contamination, and carries implications for treatment, follow-up, and long-term morbidity ${ }^{7,8}$. The National Institute for Health and Clinical Excellence (NICE) guidelines from the United Kingdom list clean catch urine (CCU) as the preferred method of urine collection for all children, unless a specimen is unobtainable by the CCU method $^{6}$. In contrast, American Academy of Pediatrics (AAP) guidelines state convenient methods can be used to obtain a specimen for urinalysis, but for patients where antibiotics for potential or suspected UTI are to be commenced, a urinary catheter or suprapubic aspirate (SPA) specimen should be obtained for reliable diagnosis ${ }^{5}$. In younger children, both NICE and AAP guidelines recommend all urine samples should be sent for culture for definitive diagnosis of UTI.

$\mathrm{CCU}$ is often favored for being less invasive and requiring less technical expertise than SPA and catheter methods, in both hospital and community based settings ${ }^{9}$. However, contamination rates in CCU are higher than by SPA or catheter collection ${ }^{10}$, ranging from $16-38 \%{ }^{10-14}$. There is significant heterogeneity in how $\mathrm{CCU}$ is obtained in clinical 
practice $^{15}$ : techniques, cleaning solutions, role of collector and cleaning method vary broadly ${ }^{14,16}$. Parents report that obtaining a CCU is time consuming and messy ${ }^{17}$. One study describes simple measures to improve contamination rates with parents collecting $\mathrm{CCU}^{15}$, otherwise there is a paucity of studies analyzing technique standardization for the person collecting the specimen.

Prolonged time spent collecting CCU has potential impacts for both the clinician and patient. Waiting for CCU may tie up valuable resources in clinical settings due to its time-consuming nature, increasing patient length of stay in the emergency department (ED). As time passes, the likelihood of contamination may increase due to difficulty preventing accidental contamination of the peri-genital area or collection jar, or CCU being abandoned by the parent or clinician. There is a lack of evidence to describing how long it takes to collect CCU in pre-continent children in the clinical environment, or whether increased time to obtaining CCU correlates with increased contamination rates. A number of novel techniques have been suggested to speed urine collection in an attempt to address these concerns ${ }^{18-22}$.

The aim of this study was to evaluate the success and time taken for urine collection by $\mathrm{CCU}$, and to investigate if there is a relationship between the time taken to collection and contamination rates. We plan to characterize the diagnostic yield of CCU in diagnosing or excluding UTI by culture in the paediatric ED setting.

\section{METHODS}




\section{Study design, participants and setting}

This prospective observational study was performed at the Royal Children's Hospital $(\mathrm{RCH})$ Melbourne ED. $\mathrm{RCH}$ is a large tertiary paediatric hospital in Australia with 82,000 ED presentations per year. Recruitment was conducted between July and November 2014. The RCH Hospital Ethics committee approved the study (HREC 34085D).

Pre-continent children aged between 2-48 months, where the treating clinician determined a urine specimen was required, were prospectively recruited following informed verbal consent in a convenience sample. Standardized packs were available at the two main ED staff bases, containing sterile water, gauze, urine jar, timer, consent and data collection forms. ED clinicians were provided with education about the study at staff meetings, clinical handovers and via posters in the ED. A total of 93 nurses and doctors recruited participants for the study. CCU was performed as per $\mathrm{RCH}$ clinical guidelines ${ }^{23}$, with initial cleaning of the peri-genital area with sterile water and the parent or carer provided with a sterile container to catch a urine sample. Children able to void on request, or who required an immediate urine sample via another method, were excluded. Data was collected on demographic information, related clinical parameters, clinical indication (see Table 1), outcome of collection attempts and the time taken for collection attempts and voiding. If data recording was incomplete, investigators approached the treating clinicians for clarification or searched medical records for relevant clinical details where possible.

[table 1] 
Urine culture outcomes were determined from hospital laboratory data once results were available. Data from the case record form were entered into Excel (Microsoft 2011) and verified by a second investigator before analyses using STATA 14.0 (StataCorp. 2015. Stata Statistical Software: Release 14. College Station, TX: StataCorp LP). The study followed the STROBE reporting guidelines for observational studies ${ }^{24}$.

\section{Definitions}

The doctor or nurse started the timer when they removed the nappy at the bedside, before commencing cleaning of the peri-genital area. The timer was stopped with any of the following outcomes: 1) "successful catch" = child voided and urine collected in specimen jar. 2) "missed catch" = child voided but urine was not collected in specimen jar. 3) "stopped" = the CCU collection attempt was ceased for any other reason (e.g. change in clinician preference for collection method, urine sample no longer required or attempt was abandoned for other reasons).. The time taken for $\mathrm{CCU}$ and outcome was recorded for each attempt per child, if more than one attempt occurred in the ED.

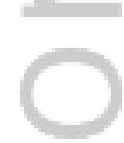

Urine culture results were defined according to hospital laboratory microbiology guidelines for clean catch urine using a $10 \mu \mathrm{L}$ loop on Horse Blood Agar. Contamination was defined by a urine culture reported as 'mixed growth', if there were e 2 colony types with Colony Forming Units (CFU) e 10,000/ml, or e 3 colony types with CFU e $1,000 / \mathrm{ml}$, or where the predominant organism was a known contaminant (e.g skin or vaginal flora). UTI was diagnosed when a single uropathogen with CFU e 100,000/ml was present, a level comparable to other guidelines ${ }^{6}$. Urine culture with intermediate 
CFU growth less than these cutoffs were designated 'no significant growth', with repeat collection recommended if clinically indicated.

\section{Analysis}

The sample size was based on the estimated proportion of children voiding within 5 minutes of initiation of the CCU attempt. This was regarded as the most important value to determine, as it would be the basis for future interventional studies ${ }^{21,22,25}$. We wanted to determine the voiding rate within $+/-5 \%$ of the actual value, which was estimated at around $10 \%$; this required the enrolment of approximately 200 patients.

Comparative analysis was conducted for results of the proportion of voids within 5 minutes, and first attempt of CCU collection for each child (time spent collecting, outcome and contamination status). Time spent collecting was summarised by median, and interquartile range (IQR) as its distribution was skewed. Outcome and contamination status were summarised by percentages with $95 \%$ confidence intervals. Chi-square test was used to compare proportions between groups.

\section{RESULTS}

During the study period 245 participants were enrolled. Twenty-seven were subsequently excluded (17 did not record primary outcome, 7 were outside of age limits, 3 had no identifying information on study form, 1 outlier with collection duration of 540 minutes) leaving 217 eligible for study inclusion and analysis (see Figure 1). Eighty-six participants (40\%) were female, median age was 10 months (IQR 6 - 18 months). 
Specimens were obtained mainly for work-up of fever without focus or other clinical findings predominantly attributable to UTI (Table 1). Twelve per cent of participants had a past history of UTI.

In total there were $247 \mathrm{CCU}$ collection attempts from 217 children, 28 children had two separate collection attempts and 2 children had three attempts during their ED presentation. Of the 217 children, 160 (74\%) ultimately had successful CCU collection, $22(10 \%)$ had urine subsequently collected by an invasive method, and $31(14 \%)$ had urine collection abandoned. From the $160 \mathrm{CCU}$ specimens obtained, 129 had urine culture performed of which 50 were contaminated (39\%). Ten children (8\%) had a positive urine culture result of a single pathogen suggesting a diagnosis of UTI.

For the first CCU attempt, the median time spent collecting urine was 30.5 minutes (IQR 11-66). The median time taken when CCU was “stopped" was 71 minutes (IQR 42.5-93), compared with when "successful" 25 minutes (IQR 7-46.5) or "missed" 27 minutes (IQR11.6 - 59). The outcome of the first CCU attempt was "successful" in 64\% (95\% CI: $58-70 \%)$, "missed" in 16\% (95\% CI: $11-20 \%)$ and "stopped" in 20\% (95\% CI: 15 $26 \%$ ). The likelihood of obtaining successful urine collection decreased after one hour of attempted CCU (see Figure 2). Twenty-seven of 217 children (12\%, 95\% CI 8 - 18\%) passed urine within 5 minutes at first CCU attempt. In the younger age group, our study found 16 of 114 infants $2-12$ months (14\%, 95\% CI 8 - 22\%) passed urine within 5 minutes.

This article is protected by copyright. All rights reserved. 
There was no difference in contamination by time to successful urine collection. Figure 3 shows that the proportion of contamination by interval of time to successful urine collection does not vary, and confidence intervals of proportions overlap.

Females had a higher proportion of contaminated CCU than males, $53 \%$ vs $32 \%$ $(\mathrm{p}=0.02)$ (Table 2). Contamination was highest in the youngest age group (2-6 months). Further analyses of time of last feed and combined collecting time where there were multiple attempts were not statistically significant for contamination.

[table 2]

This article is protected by copyright. All rights reserved. 


\section{DISCUSSION}

This study prospectively assessed the time taken and success of attempted CCU collection in pre-continent children. The indication for the majority of specimens was investigation of fever without focus, symptoms attributable to UTI, or clinical suspicion of UTI. Our findings indicate that CCU attempts can be time consuming, and only $12 \%$ voided within 5 minutes. $64 \%$ of first CCU attempts were successful, and as time passes beyond an hour the likelihood of successfully obtaining CCU plateaued. Three-quarters of patients who voided for successful CCU did so within 46 minutes, and the median time was similar for "successful" (25 minutes) and "missed" (27 minutes) CCU attempts.

Clinicians will need to consider these results when they plan to collect a CCU sample. They challenge the role of CCU as the collection method of choice for the investigation of suspected UTI in young children due to the time required, and the proportion of missed and unsuccessful attempts requiring re-collection. These findings suggest that in practice, CCU attempts should be time limited and alternate collection methods considered beyond one hour. The rate of spontaneous void at 5 minutes will provide an important baseline value and starting point for sample size calculation for studies attempting to speed CCU in young children.

When culture was performed, a high proportion (39\%) were contaminated. There was a significantly higher contamination rate in females, and a trend towards higher contamination in younger infants (2-6 months). In this cohort of patients, the overall 
proportion of successfully collected, uncontaminated urine culture obtained from a CCU was 79/217 (36\%), although not all specimens collected were cultured.

When embarking on a CCU attempt, we estimate that if all urine specimens successfully collected were sent for culture (160 of $217,74 \%$ ), with a contamination rate of $39 \%$ (uncontaminated specimen rate of $61 \%$ ), a clinician could expect a $45 \%$ chance of obtaining an uncontaminated specimen from this method $(74 \% \times 61 \%=45 \%)$.

This study found no clear relationship between the time taken for collection and CCU contamination. Our hypothesis that contamination is related to time taken for collection arose from clinical observations that accidental contact of the specimen jar with the perigenital area after cleaning was common. Furthermore, we hypothesized that such contact was more likely to occur with the passage of time. An explanation for these findings may be that the contamination occurs from commensal organisms present at the distal urethra, with the contact of urine passing the vulva, vagina or labia in girls; or prepuce in boys. This explanation is supported by the ballooning of the prepuce and vaginal reflux seen in a proportion of children ( $38 \%$ boys and $14.3 \%$ of girls) during voiding on fluoroscopic micturating cystourethrograms ${ }^{26}$. The recommended collection approach in adults, is that the first void is discarded before obtaining a "mid-stream" collection. Considering the difficulties obtaining CCU in young children, a definite "midstream" specimen is generally difficult to achieve. Studies on reducing contamination rates in CCU by cleaning are mixed, with some showing external cleaning does not have a significant impact on contamination rates ${ }^{27,28}$ whilst a recent systematic review suggests 
that if non-invasive urine collection is being considered for young children, midstream collection with cleansing is recommended ${ }^{29}$.

There is no consensus definition of contamination for clean catch urine culture in children. In published studies where a definition is outlined, numbers of organisms and CFU cut-offs vary ${ }^{12,14,19,29-31}$. The definition used in this study was determined by the hospital microbiology laboratory, which stratifies interpretation of culture growth and contamination depending on method of collection, number of organisms and CFU, similar to recommended UK standards for microbiology investigations ${ }^{32}$. The contamination rate found in this study is comparable with rates reported in other studies of $\mathrm{CCU}^{10-14}$. Interpretation of microbiological culture results with growth at lower $\mathrm{CFU}$ should be interpreted in the clinical context, the presence or absence of pyuria, and squamous epithelial cells on microscopy, and may represent early UTI, contamination or asymptomatic bacteriuria. .

This study has some limitations. It relied on opportunistic recruiting by ED clinicians rather than consecutive recruitment, and was conducted in a single tertiary paediatric ED setting. A proportion (19\%) of successfully caught urine specimens collected were not sent for laboratory culture and therefore contamination of these specimens could not be determined. This may be due to the urine dipstick results being deemed sufficient for clinical decision-making, despite urine culture being the recommended test for diagnosing UTI in young children ${ }^{4-6}$. We did not measure the preparation time taken prior to commencement of attempt (e.g. whilst encouraging oral intake), or time between 
attempts when more than one attempt was required. As the clinician was responsible for starting the timer as well as commencing cleaning, a small degree of uncertainty of the measurement is possible. Whilst the majority of specimens were obtained to investigate for UTI, the clinical course may have changed due to interventions (e.g. fluids, treatment, other investigations, observation), changing the clinician's ongoing decision regarding urine collection. Clinicians were aware contamination outcomes were being recorded and this may have influenced cleaning behavior, however contamination rates are similar to previous reports from this $\mathrm{ED}^{10}$.

An ideal method of reducing contamination rates and obtaining $\mathrm{CCU}$ in a timely manner remains elusive. There is promising evidence for stimulation methods that hasten $\mathrm{CCU}$ collection, defining successful collection as a sample obtained within 5 minutes $^{19,20,33}$. However two of these studies are limited to the Neonatal Intensive Care setting, samples were mostly obtained for reasons other than fever or suspected UTI, and patients with poor feeding and dehydration were excluded, limiting the generalizability of these findings ${ }^{19,20}$.

Excessive time waiting for CCU may have a negative impact on families and clinical resources, particularly where CCU collection is ultimately unsuccessful and subsequent progression to invasive collection methods is required. Fourteen percent of urine collection attempts were abandoned entirely, presumably due to identification of an alternate focus or improved clinical state in the period of time waiting for CCU. The 
clinician should be aware of the expected contamination rate of CCU, and consider this when selecting the method of urine collection.

\section{CONCLUSION}

This study provides an important perspective for clinicians to consider when considering how to collect a urine specimen from young children, and highlights several limitations of the CCU method. CCU attempts are time consuming, and have a low rate of diagnostic success for the investigation of UTI with high rates of missed catches and contamination. Our data would suggest that waiting for a CCU beyond an hour is unlikely to be successful: at this time point the ED clinician might well choose to change collection strategy.

Financial Disclosure: No financial relationships relevant to this article to disclose. Conflict of Interest: No conflicts of interest to disclose. Ethics approval: APPROVED (HREC 34085D)

Clinical Trial Registration: Nil

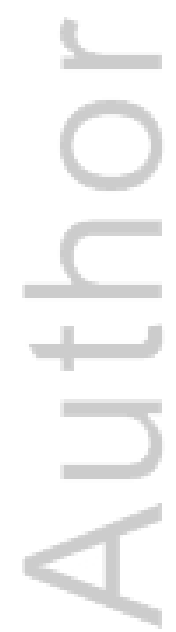

This article is protected by copyright. All rights reserved. 


\section{References:}

1. Hoberman A, Wald ER. Urinary tract infections in young febrile children. The Pediatric infectious disease journal. 1997;16(1):11-17.

2. Shaikh N, Morone NE, Bost JE, Farrell MH. Prevalence of urinary tract infection in childhood: a meta-analysis. The Pediatric infectious disease journal. 2008;27(4):302-308.

3. Haddon RA, Barnett PL, Grimwood K, Hogg GG. Bacteraemia in febrile children presenting to a pae3iatric emergency department. Med J Aust. 1999;170(10):475-478.

4. Royal Childrens Hospital Melbourne. Febrile Child Guideline. available at http://www.rch.org.au/clinicalguide/guideline index/Febrile Child/ Last accessed July 2015.

5. Subcommittee on Urinary Tract Infection SCoQIM, Roberts KB. Urinary tract infection: clinical practice guideline for the diagnosis and management of the initial UTI in febrile infants and children 2 to 24 months. Pediatrics. 2011;128(3):595-610.

6. National Collaborating Centre for Women's Children's Health. National Institute for Health and Clinical Excellence: Guidance. Urinary Tract Infection in Children: Diagnosis, Treatment and Long-term Management. 2007.

7. Ransley PG, Risdon RA. Reflux nephropathy: effects of antimicrobial therapy on the evolution of the early pyelonephritic scar. Kidney international. 1981;20(6):733-742.

8. Becker GJ, Kincaid-Smith P. Reflux nephropathy: the glomerular lesion and progression of renal failure. Pediatric nephrology. 1993;7(4):365-369.

9. Buntsma D, Stock A, Bevan C, Babl FE, Paediatric Research in Emergency Departments International C. How do clinicians obtain urine samples in young children? Emergency medicine Australasia : EMA. 2012;24(1):118-119.

10. Tosif S, Baker A, Oakley E, Donath S, Babl FE. Contamination rates of different urine collection methods for the diagnosis of urinary tract infections in young children: an observational cohort study. J Paediatr Child Health. 2012;48(8):659-664.

11. Altuntas N, Celebi Tayfur A, Kocak M, Razi HC, Akkurt S. Midstream cleancatch urine collection in newborns: a randomized controlled study. European Journal of Pediatrics. 2014;174(5):577-582.

12. Labrosse M, Levy A, Autmizguine J, Gravel J. Evaluation of a New Strategy for Clean-Catch Urine in Infants. Pediatrics. 2016;138(3).

13. Tran A, Fortier C, Giovannini-Chami L, et al. Evaluation of the Bladder Stimulation Technique to Collect Midstream Urine in Infants in a Pediatric Emergency Department. PLoS One. 2016;11(3):e0152598.

14. Vaillancourt S, McGillivray D, Zhang X, Kramer MS. To clean or not to clean: effect on contamination rates in midstream urine collections in toilet-trained children. Pediatrics. 2007;119(6):e1288-1293. 
15. Giddens J, Robinson G. How accurately do parents collect urine samples from their children? A pilot study in general practice. Br J Gen Pract. 1998;48(427):987-988.

16. Hardy JD, Furnell PM, Brumfitt W. Comparison of sterile bag, clean catch and suprapubic aspiration in the diagnosis of urinary infection in early childhood. British journal of urology. 1976;48(4):279-283.

17. Liaw LC, Nayar DM, Pedler SJ, Coulthard MG. Home collection of urine for culture from infants by three methods: survey of parents' preferences and bacterial contamination rates. Bmj. 2000;320(7245):1312-1313.

18. Davies P, Greenwood R, Benger J. Randomised trial of a vibrating bladder stimulator-the time to pee study. Archives of disease in childhood. 2008;93(5):423-424.

19. Altuntas N, Tayfur AC, Kocak M, Razi HC, Akkurt S. Midstream clean-catch urine collection in newborns: a randomized controlled study. European journal of pediatrics. 2015;174(5):577-582.

20. Herreros Fernandez ML, Gonzalez Merino N, Tagarro Garcia A, et al. A new technique for fast and safe collection of urine in newborns. Archives of disease in childhood. 2013;98(1):27-29.

21. Kaufman J, Tosif S, Fitzpatrick P, et al. Quick-Wee: a novel non-invasive urine collection method. Emergency medicine journal : EMJ. 2017;34(1):63-64.

22. Kaufman J, Fitzpatrick P, Tosif S, et al. The QuickWee trial: protocol for a randomised controlled trial of gentle suprapubic cutaneous stimulation to hasten non-invasive urine collection from infants. BMJ Open. 2016;6(8).

23. Royal Childrens Hospital Melbourne. Urinary Tract Infection Guideline. Last accessed June 2016;

http://www.rch.org.au/clinicalguide/guideline index/Urinary Tract Infectio n Guideline/.

24. von Elm E, Altman DG, Egger M, et al. The Strengthening the Reporting of Observational Studies in Epidemiology (STROBE) statement: guidelines for reporting observational studies. J Clin Epidemiol. 2008;61(4):344-349.

25. Kaufman J, Fitzpatrick P, Tosif S, et al. Faster clean catch urine collection from infants: the Quick-Wee randomised controlled trial. BMJ. 2017.

26. Tullus K, Hooman N, Easty M. Flushing of the vagina and the prepuce-a cause for contaminated urine cultures in children. Pediatric nephrology (Berlin, Germany). 2016.

27. Leisure MK, Dudley SM, Donowitz LG. Does a Clean-Catch Urine Sample Reduce Bacterial Contamination? New England Journal of Medicine. 1993;328(4):289-290.

28. Lifshitz E, Kramer L. Outpatient urine culture: Does collection technique matter? Archives of Internal Medicine. 2000;160(16):2537-2540.

29. LaRocco MT, Franek J, Leibach EK, et al. Effectiveness of Preanalytic Practices on Contamination and Diagnostic Accuracy of Urine Cultures: a Laboratory Medicine Best Practices Systematic Review and Meta-analysis. Clinical microbiology reviews. 2016;29(1):105-147.

30. Liaw LCT, Nayar DM, Pedler SJ, Coulthard MG. Home collection of urine for culture from infants by three methods: survey of parents' preferences and

This article is protected by copyright. All rights reserved. 
bacterial contamination rates. BMJ : British Medical Journal.

2000;320(7245):1312-1313.

31. Verliat-Guinaud J, Blanc P, Garnier F, Gajdos V, Guigonis V. A midstream urine collector is not a good alternative to a sterile collection method during the diagnosis of urinary tract infection. Acta paediatrica (Oslo, Norway : 1992). 2015;104(9):e395-400.

32. England SUPH. UK Standards for Microbiology Investigations: Investigation of urine. Bacteriology, B 41 Issue no: 81, . 2016.

33. Herreros ML, Tagarro A, García-Pose A, Sánchez A, Cañete A, Gili P. Accuracy

= of a new clean-catch technique for diagnosis of urinary tract infection in infants younger than 90 days of age. Paediatrics \& Child Health (1205-7088). $2015 ; 20(6)$.

This article is protected by copyright. All rights reserved. 


\begin{tabular}{|c|c|c|}
\hline & Indication & $\mathbf{N}(\%)$ \\
\hline & Fever without focus & $127(59 \%)$ \\
\hline & Vomiting & $31(14 \%)$ \\
\hline & Clinically suspected UTI & $28(13 \%)$ \\
\hline & Other & $9(4 \%)$ \\
\hline & Failure to thrive & $6(3 \%)$ \\
\hline & Irritability & $6(3 \%)$ \\
\hline & Oedema/Proteinuria & $3(1 \%)$ \\
\hline & Reduced oral intake & $3(1 \%)$ \\
\hline & Not specified & $3(1 \%)$ \\
\hline 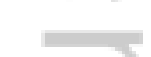 & Jaundice & $1(0.5 \%)$ \\
\hline
\end{tabular}

Table 1: Clinician indication for obtaining Clean Catch Urine

This article is protected by copyright. All rights reserved. 


\begin{tabular}{|c|c|c|c|c|c|}
\hline Variable & $\mathbf{n}$ & $\begin{array}{c}\text { Contaminated } \% \\
\text { (n) }\end{array}$ & \multicolumn{2}{|c|}{$\begin{array}{l}\text { 95\% Confidence } \\
\text { interval }\end{array}$} & $\mathbf{p}$ \\
\hline Female & 47 & $53 \%(25)$ & $39 \%$ & $67 \%$ & \\
\hline Male & 63 & $32 \%(20)$ & $20 \%$ & $43 \%$ & 0.02 \\
\hline Difference & & $21 \%$ & $3 \%$ & $40 \%$ & \\
\hline \multicolumn{6}{|l|}{$\begin{array}{l}\text { Age group } \\
\text { (months) }\end{array}$} \\
\hline $2-6$ & 37 & $57 \%(21)$ & $41 \%$ & $73 \%$ & \multirow{4}{*}{0.10} \\
\hline $6-12$ & 39 & $36 \%(14)$ & $21 \%$ & $51 \%$ & \\
\hline $12-24$ & 22 & $27 \%(6)$ & $9 \%$ & $46 \%$ & \\
\hline$>24$ & 12 & $33 \%(4)$ & $7 \%$ & $60 \%$ & \\
\hline
\end{tabular}

Table 2: Proportion of total CCU contaminated, by sex and age group

This article is protected by copyright. All rights reserved. 


\section{University Library}

\section{- M M N E R VA A gateway to Melbourne's research publications}

Minerva Access is the Institutional Repository of The University of Melbourne

Author/s:

Tosif, S;Kaufman, J;Fitzpatrick, P;Hopper, SM;Hoq, M;Donath, S;Babl, FE

Title:

Clean catch urine collection: Time taken and diagnostic implication. A prospective observational study

Date:

2017-10-01

Citation:

Tosif, S., Kaufman, J., Fitzpatrick, P., Hopper, S. M., Hoq, M., Donath, S. \& Babl, F. E. (2017). Clean catch urine collection: Time taken and diagnostic implication. A prospective observational study. JOURNAL OF PAEDIATRICS AND CHILD HEALTH, 53 (10), pp.970-975. https://doi.org/10.1111/jpc. 13595.

Persistent Link:

http://hdl.handle.net/11343/293036 